\title{
The medical food Souvenaid affects brain phospholipid metabolism in mild Alzheimer's disease: results from a randomized controlled trial
}

Anne Rijpma ${ }^{1,2}$, Marinette van der Graaf ${ }^{3,4}$, Marieke M. Lansbergen ${ }^{5}$, Olga Meulenbroek ${ }^{1,2}$, Aysun Cetinyurek-Yavuz ${ }^{5}$, John W. Sijben ${ }^{5}$, Arend Heerschap ${ }^{3+}$ and Marcel G. M. Olde Rikkert ${ }^{12^{*+}}$

\begin{abstract}
Background: Synaptic dysfunction contributes to cognitive impairment in Alzheimer's disease and may be countered by increased intake of nutrients that target brain phospholipid metabolism. In this study, we explored whether the medical food Souvenaid affects brain phospholipid metabolism in patients with Alzheimer's disease.

Methods: Thirty-four drug-naive patients with mild Alzheimer's disease (Mini Mental State Examination score $\geq 20$ ) were enrolled in this exploratory, double-blind, randomized controlled study. Before and after 4-week intervention with Souvenaid or an isocaloric control product, phosphorus and proton magnetic resonance spectroscopy (MRS) was performed to assess surrogate measures of phospholipid synthesis and breakdown (phosphomonoesters [PME] and phosphodiesters [PDEs]), neural integrity ( $\mathrm{N}$-acetyl aspartate), gliosis (myo-inositol), and choline metabolism (cholinecontaining compounds [tCho]). The main outcome parameters were PME and PDE signal intensities and the PME/PDE ratio.

Results: MRS data from 33 patients (60-86 years old; $42 \%$ males; Souvenaid arm $n=16$; control arm $n=17$ ) were analyzed. PME/PDE and tCho were higher after 4 weeks of Souvenaid compared with control (PME/PDE least squares [LS] mean difference [95\% Cl] 0.18 [0.06-0.30], $p=0.005$; tCho LS mean difference [95\% Cl] 0.01 [0.00-0.02], $p=0.019$ ). No significant differences were observed in the other MRS outcome parameters.
\end{abstract}

Conclusions: MRS reveals that Souvenaid affects brain phospholipid metabolism in mild Alzheimer's disease, in line with findings in preclinical studies.

Trial registration: Netherlands Trial Register, NTR3346. Registered on 13 March 2012.

Keywords: Nutrition, Phospholipid metabolism, Magnetic resonance spectroscopy, ${ }^{31} \mathrm{P}-\mathrm{MRS},{ }^{1} \mathrm{H}-\mathrm{MRS}$, Medical food, Alzheimer's disease, Dementia, Souvenaid, Fortasyn Connect

\section{Background}

Synaptic dysfunction is a major contributing factor to cognitive impairment in Alzheimer's disease (AD) $[1,2]$ and may be caused by deficits in neuronal membrane composition and function [3, 4]. Because the neuronal membrane is composed mainly of phospholipids [5], interventions that

\footnotetext{
* Correspondence: marcel.olderikkert@radboudumc.nl

${ }^{\dagger}$ Equal contributors

${ }^{1}$ Department of Geriatric Medicine, Radboud University Medical Center, P.O. Box 9101, 6500 HB Nijmegen, The Netherlands

${ }^{2}$ Radboudumc Alzheimer Center, Donders Institute for Brain, Cognition and Behavior, Radboud University Medical Center, Nijmegen, The Netherlands Full list of author information is available at the end of the article
}

target brain phospholipid metabolism may affect cognitive function in $\mathrm{AD}$.

The most abundant phospholipids in the neuronal membrane are phosphatidylethanolamine (PE) and phosphatidylcholine (PC) $[4,5]$. They are formed in the Kennedy cycle, wherein phosphomonoesters (PMEs) are converted to phospholipids that can then be incorporated into neuronal membranes [6]. The breakdown of these phospholipids releases phosphodiesters (PDEs), which can either be used for resynthesis or broken down further [7]. The synthesis of brain phospholipids is influenced by the availability of specific nutrients to the brain, consisting of 
rate-limiting phospholipid precursors $[8,9]$. This is affected not only by nutritional intake but also by the intake of cofactors that influence precursor uptake and metabolism. Increasing the availability of several precursors proved to have a synergistic effect on phospholipid formation as well as on dendritic spine density [9-12]. Furthermore, circulating levels of most of these nutrients (precursors and cofactors) as well as brain choline levels are lower in patients with $\mathrm{AD}$ [13-15], thus lowering precursor availability. The specific multinutrient combination Fortasyn ${ }^{\circ}$ Connect (FC) in the medical food Souvenaid ${ }^{\circ}$ (Nutricia Advanced Medical Nutrition, Utrecht, The Netherlands) contains precursors and cofactors in the phospholipid synthesis pathway (docosahexaenoic acid [DHA]; eicosapentaenoic acid [EPA]; uridine monophosphate [UMP]; choline; phospholipids; selenium; folic acid; and vitamins $\mathrm{B}_{6}, \mathrm{~B}_{12}, \mathrm{C}$, and $\mathrm{E}$ ), and it has been formulated to promote neuronal membrane (phospholipid) formation and function in $\mathrm{AD}$ [8]. Previous studies in animal models of $\mathrm{AD}$ and aging have shown that long-term supplementation with FC positively affects exploratory behavior and memory [16, 17], in addition to enhancing phospholipid synthesis and improving cholinergic transmission [18]. This indicates that supporting synaptic function by increasing phospholipid formation is a promising strategy to improve cognition or reduce cognitive decline.

Randomized controlled trials with patients with AD demonstrated improvement in memory performance in those with mild AD over 12-24 weeks of intervention with this specific multinutrient combination $[19,20]$ as well as altered functional connectivity and preserved brain network organization $[20,21]$. However, the physiological underpinnings in humans remain to be further elucidated. The present study was designed to provide more insight into the hypothesized underlying mechanisms of FC (i.e., promoting neuronal membrane [phospholipid] formation and function) in humans.

Neither synapse number nor phospholipid membrane composition can be assessed directly in vivo, but phosphorus magnetic resonance spectroscopy $\left({ }^{31} \mathrm{P}-\mathrm{MRS}\right)$ allows for the noninvasive investigation of phospholipid building blocks and breakdown products (i.e., PME and PDE, respectively) [22]. In addition, using proton $\left({ }^{1} \mathrm{H}\right)$ MRS, brain metabolites related to neural integrity ( $N$-acetylaspartate [NAA]), gliosis (myo-inositol [mI]), choline metabolism (total choline [tCho]), and energy metabolism (total creatine $[\mathrm{tCr}])$ can be assessed as well [22, 23]. Hence, in the present study, we explored whether the medical food Souvenaid affects brain phospholipid metabolism and neural integrity in patients with mild AD using ${ }^{31} \mathrm{P}-\mathrm{MRS}$ and ${ }^{1} \mathrm{H}$-MRS.

\section{Methods}

\section{Subjects and design}

The MRS AD study was a 4-week, randomized, controlled, double-blind, single-center trial with patients with mild
AD. Significant effects of FC on memory performance were reported in this population previously $[19,20]$. In the present study, we explored whether FC affects brain phospholipid metabolism in patients with mild AD. All visits took place between October 2012 and February 2015 at the Radboud University Medical Center (Nijmegen, The Netherlands). AD drug-naive patients aged $\geq 50$ years with a diagnosis of probable or possible $\mathrm{AD}$ (according to the revised 2011 criteria of the National Institute of Neurological and Communicative Disorders and Stroke and Alzheimer's Disease and Related Disorders Association [24]) with evidence of the pathophysiological process (i.e., from structural magnetic resonance imaging [MRI] or cerebrospinal fluid [CSF] biomarker assays), a Mini Mental State Examination (MMSE) score $\geq 20$, and a Geriatric Depression Scale (GDS) score $\leq 6$ (of 15) were recruited from the hospital's memory clinic or by referral from regional hospitals. All subjects were drug-naive for $\mathrm{AD}$ medication (cholinesterase inhibitors and $\mathrm{N}$-methyl-D-aspartate antagonists) and were free of neurological or psychiatric disorders (other than dementia). To increase subject recruitment, eligibility criteria were extended during the study to include patients who were off $\mathrm{AD}$ medication for at least 3 months prior to the study, but no additional subjects were recruited who did not meet the original criteria. Subjects did not consume oily fish more than twice per week or use nutritional supplements containing DHA, EPA within 2 months prior to baseline, or nutritional supplements containing $>200 \%$ of the recommended daily allowance of vitamins $\mathrm{B}_{6}(2.8 \mathrm{mg}), \mathrm{B}_{12}(5 \mu \mathrm{g}), \mathrm{C}(160$ $\mathrm{mg}), \mathrm{E}(24 \mathrm{mg})$, or folic acid $(0.4 \mathrm{mg})$ within 1 month prior to baseline. Use of anticholinergic or antipsychotic medication and of other medical foods or investigational products was also prohibited within 1 month prior to baseline, as well as changes in dose of lipid-lowering medication, antihypertensives, and/or antidepressants. All subjects were screened for MRI contraindications before inclusion in the study. Written informed consent was obtained from all patients and their informal caregivers. The local ethics committee reviewed and approved the protocol. The study was conducted in accordance with the Declaration of Helsinki and is registered in the Netherlands Trial Register (NTR3346).

Medical history, medication and nutritional supplement use, MMSE score, GDS score, date of birth, sex, ethnicity, smoking habits, alcohol consumption, and family history of $\mathrm{AD}$ were recorded for all subjects at the first (screening) visit. Eligible subjects were randomly allocated to receive either the test product (i.e., Souvenaid containing the specific nutrient combination FC) or an isocaloric control product once daily as a drink for a double-blind period of 4 weeks (1:1 randomization, stratified on the basis of sex). The test product contained DHA; EPA; phospholipids; choline; UMP; selenium; folic acid; and vitamins $B_{6}, B_{12}$, $C$, and $E$ (Table 1). On the basis of previous publications in which ${ }^{31} \mathrm{P}-\mathrm{MRS}$ brain metabolites in humans were already affected 
Table 1 Nutritional composition of the study products (amount per daily dose of $125 \mathrm{ml}$ )

\begin{tabular}{lll}
\hline & Control & Active \\
\hline Energy & $125 \mathrm{kcal}$ & $125 \mathrm{kcal}$ \\
Protein & $3.8 \mathrm{~g}$ & $3.8 \mathrm{~g}$ \\
Carbohydrate & $16.5 \mathrm{~g}$ & $16.5 \mathrm{~g}$ \\
Fat & $4.9 \mathrm{~g}$ & $4.9 \mathrm{~g}$ \\
EPA & 0 & $300 \mathrm{mg}$ \\
DHA & 0 & $1200 \mathrm{mg}$ \\
Phospholipids & 0 & $106 \mathrm{mg}$ \\
Choline & 0 & $400 \mathrm{mg}$ \\
UMP & 0 & $625 \mathrm{mg}$ \\
Vitamin E (a-tocopherol equivalents) & 0 & $40 \mathrm{mg}$ \\
Vitamin C & 0 & $80 \mathrm{mg}$ \\
Selenium & 0 & $60 \mu \mathrm{g}$ \\
Vitamin $B_{12}$ & 0 & $3 \mu \mathrm{g}$ \\
Vitamin $B_{6}$ & 0 & $1 \mathrm{mg}$ \\
Folic acid & 0 & $400 \mu \mathrm{gg}$ \\
\hline
\end{tabular}

EPA Eicosapentaenoic acid, DHA Docosahexaenoic acid, UMP

Uridine monophosphate

Test product is Souvenaid, containing the specific nutrient combination Fortasyn Connect. Souvenaid and Fortasyn are registered trademarks of Nutricia N.V

after 1-week administration of uridine [25] and 6-week administration of citicoline [26], it was expected that an intervention period of 4 weeks was sufficient to observe an effect of the test product on ${ }^{31} \mathrm{P}$-MRS brain metabolites.

Numbered and sealed randomization envelops (containing one of four codes, two representing the test group and two the control group) were generated at Nutricia Research by the clinical studies supplies manager and opened upon randomization on-site by the investigator. All subjects and any person involved in subject recruitment, group allocation, data acquisition and processing, or statistical analyses were blinded to the intervention group (test or control) until the analyses of the main outcome parameters were completed. The study product was packaged in tetra-packs (until 15 January 2014) or plastic bottles (from 15 January 2014 onward) and labeled with one of the four randomization codes. With assistance from their informal caregivers, subjects recorded intake of the product daily in a diary, which was used to verify intake at the week 4 visit.

At baseline and after 4 weeks, venous blood samples were taken and magnetic resonance (MR) measurements were performed. MR measurements at week 4 took place at least $2 \mathrm{~h}$ after intake of the last study product. For the purposes of compliance and safety, a phone call was conducted after 14 days of product intake. A final follow-up call was conducted 2 weeks after the last visit. Subjects were instructed to minimize intake of high-choline food on the days of the baseline and week 4 visits and to keep intake of concomitant nutritional supplements and medication stable (unless deemed necessary by their physician) during the study. Analyses of the blood samples were performed as reported previously [27].

\section{MR protocol}

MRI and MRS were performed on a MAGNETOM Trio Tim System 3-T MR system (Siemens Healthcare, Erlangen, Germany) with a dual-tuned ${ }^{1} \mathrm{H} /{ }^{31} \mathrm{P}$ volume head coil (Rapid Biomedical, Würzburg, Germany). Highresolution structural MR images were acquired with a T1weighted magnetization-prepared rapid gradient echo sequence (repetition time [TR] 2300 milliseconds, echo time [TE] 3.16 milliseconds, inversion time 1100 milliseconds, 15-degree flip angle, 176 sagittal slices, slice matrix size $256 \times 256$, slice thickness $1 \mathrm{~mm}$, voxel size $1 \times 1 \times 1 \mathrm{~mm}$, acquisition time [TA] 6:25 minutes). ${ }^{31} \mathrm{P}-\mathrm{MR}$ spectra were acquired by whole-brain 3D MR spectroscopic imaging (MRSI; TR 2000 milliseconds, 40-degree flip angle, four averages, acquisition delay 0.10 milliseconds, broadband proton decoupling applied during first half of 512-millisecond acquisition duration, field of view (FOV) $260 \times 260 \times 260$ $\mathrm{mm}$; matrix size $10 \times 10 \times 10$, TA 13:08 minutes). $k$-Space was sampled with a weighted elliptical phase-encoding scheme with four averages. The FOV was centered on the midline and parallel to the line from the anterior commissure to the posterior commissure. Spatial postprocessing consisted of zero-filling to a matrix size of $16 \times 16 \times 16$ followed by spatial Fourier transformation. The nominal volume of the selected cubic voxels is about $17.5 \mathrm{~cm}^{3}$; because of the spatial response function, the spectra have contributions from a spherical area with an effective volume of about $40 \mathrm{~cm}^{3}$ at $64 \%$ of this function [28].

${ }^{1} \mathrm{H}$-MR spectra of the hippocampus were acquired with a single-slice 2D semilocalization by adiabatic selective refocusing (sLASER) MRSI sequence, with water suppression enhanced through T1 effects (TR 2100 milliseconds, TE = 30 milliseconds, 90-degree excitation flip angle, six averages, FOV $120 \times 160 \times 10 \mathrm{~mm}$, nominal voxel size $3.75 \times$ $5 \times 10 \mathrm{~mm}$ ), positioned parallel to the hippocampi. ${ }^{1} \mathrm{H}-\mathrm{MR}$ spectra of the anterior and posterior cingulate cortices (ACC and PCC, respectively) were acquired with a singlevoxel point resolved spectroscopy (PRESS) sequence with chemical shift selective water suppression (TR 3000 milliseconds, TE 30 milliseconds, 90-degree excitation flip angle, 64 averages, voxel size $27 \times 15 \times 15 \mathrm{~mm}$ [ACC] and $20 \times$ $20 \times 16 \mathrm{~mm}$ [PCC]). With each sequence, an additional MR spectrum without water suppression was obtained (2D sLASER: one average, PRESS: eight averages). The complete MR protocol, including positioning of the patient, took approximately 90 minutes. Because the ${ }^{31} \mathrm{P}$-MRS quantities PME, PDE, and PME/PDE were the main outcome measures, ${ }^{31} \mathrm{P}$-MRS measurements were always performed before ${ }^{1} \mathrm{H}$-MRS measurements in case subjects failed to complete the entire scanning protocol. 


\section{MR data analysis}

Four regions of interest (ROIs) were selected for analysis from the 3D ${ }^{31} \mathrm{P}-\mathrm{MRSI}$ data: ACC, retrosplenial cortex (RSC), and left and right hippocampi (HL and HR, respectively). Voxels covering these ROIs were selected by shifting the MRSI grid in the $x, y$, and $z$ dimensions using anatomical landmarks on the T1-weighted anatomical images. From the $2 \mathrm{D}{ }^{1} \mathrm{H}$-MRSI data, two voxels positioned on the hippocampi (HL and HR, respectively) were selected for analysis, in addition to the single-voxel data from the ACC and PCC (Fig. 1a, c).

The Metabolite Report software package (Siemens Healthcare) was used for postprocessing (i.e., zero-filling, phase correction, 100-millisecond exponential filter, baseline correction) and for automatic fitting of the ${ }^{31} \mathrm{P}-\mathrm{MR}$ spectra in the time domain using prior knowledge (unpublished data Rijpma and de Graaf [29]). Eleven wellresolved resonance peaks were fitted: the phospholipid metabolites phosphoethanolamine (PEth), phosphocholine (PCh), glycerophosphoethanolamine (GPEth), and glycerophosphocholine (GPCh); the high-energy phosphorus molecules phosphocreatine (PCr); adenosine triphosphates (ATPs) $\alpha$-ATP, $\beta$-ATP, and $\gamma$-ATP; nicotinamide adenine dinucleotide $[\mathrm{NAD}(\mathrm{H})]$ and inorganic phosphate (Pi); and membrane-bound phospholipid (MP). The intensity of each metabolite resonance was expressed as a percentage area of the total phosphorus signal in the spectrum. Thus, the data are corrected for CSF content and for differences in atrophy. PME was calculated as the sum of PEth and PCh, PDE as the sum of GPEth and GPCh, and total adenosine triphosphate as the sum of $\alpha$ ATP, $\beta$-ATP, and $\gamma$-ATP. In addition, the ratios PME/PDE, PEth/GPEth, PCh/GPCh, and $\mathrm{PCr} / \mathrm{Pi}$ were computed. Intracellular $\mathrm{pH}$ was determined from the chemical shift difference between the PCr and Pi resonance peaks [30].

Both a quantitative evaluation of the fitting results and a visual quality control were performed. Quantitatively, only fits of metabolite peaks with a Cramér-Rao lower bound (CRLB) $\leq 30 \%$ were considered reliable. Qualitatively, two spectroscopists (AR and MvdG) independently checked the spectra by visual inspection of the original spectra and the fitting results. If a metabolite peak was visually present

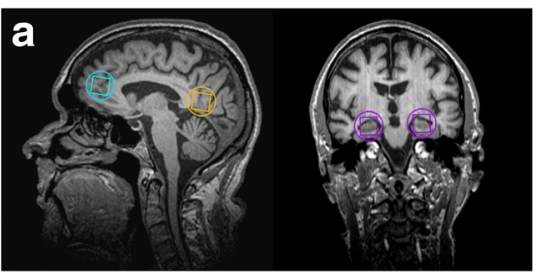

b

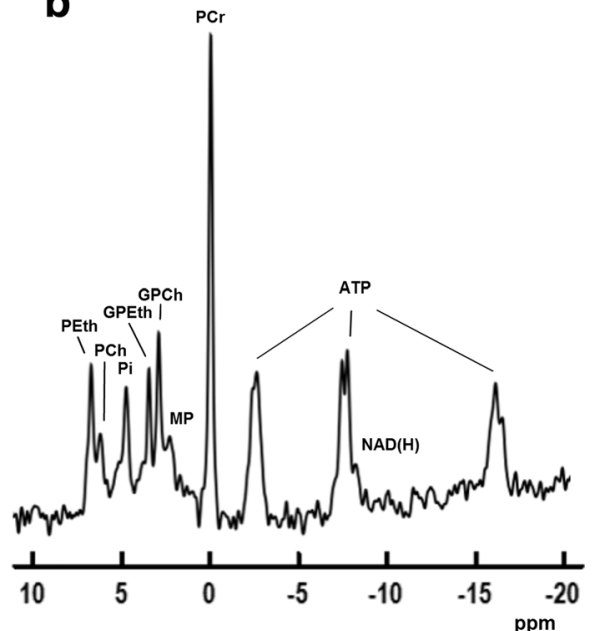

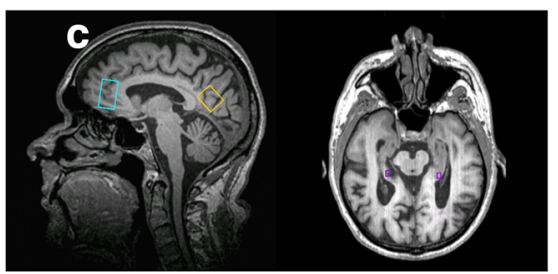

d

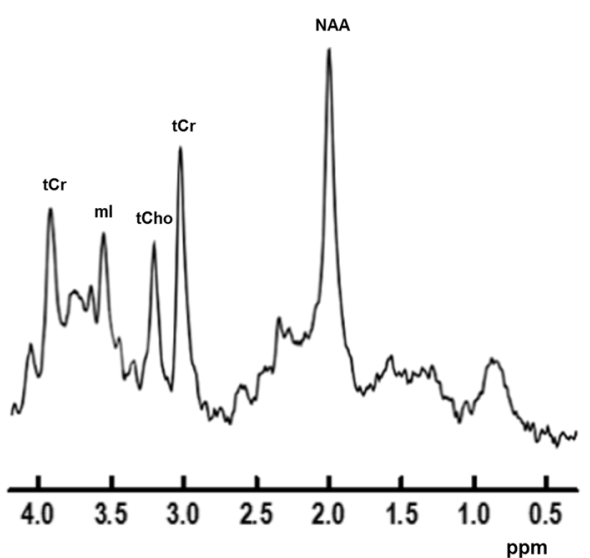

Fig. 1 Voxel selection and representative examples of ${ }^{31} \mathrm{P}$ and ${ }^{1} \mathrm{H}$ magnetic resonance (MR) spectra. a Voxel selection of ${ }^{31} \mathrm{P}-\mathrm{MR}$ spectra displayed on sagittal (left) and coronal (right) anatomical images: anterior cingulate cortex (blue), retrosplenial cortex (yellow), left and right hippocampus (purple). The nominal voxel size is indicated by squares, and the approximation of the effective spherical voxel size is indicated by circles. b Representative

${ }^{31}$ P-MR spectrum from the retrosplenial cortex. Zero-filling to 4096 data points and an 8-Hz Gaussian filter were applied. c Sagittal (left) and transversal (right) anatomical images showing single-voxel volumes of ${ }^{1} \mathrm{H}-\mathrm{MR}$ spectra in anterior cingulate cortex (blue), posterior cingulate cortex (yellow), and voxel selection of left and right hippocampi (purple). $\mathbf{d}$ Representative ${ }^{1} \mathrm{H}-\mathrm{MR}$ spectrum from the posterior cingulate cortex. Zero-filling to 8192 data points and a 2-Hz Lorentzian filter were applied. PEth, Phosphoethanolamine; PCh, Phosphocholine; Pi, Inorganic phosphate; GPEth,

Glycerophosphoethanolamine; GPCh, Glycerophosphocholine; MP, Membrane phospholipids; PCr, Phosphocreatine; ATP, Adenosine triphosphate; $\mathrm{NAD}(\mathrm{H})$, Nicotinamide adenine dinucleotide; ppm, Parts per million; NAA, N-acetyl-aspartate; tCho, Choline-containing compounds; $\mathrm{tCr}$, Total creatine; ml, Myo-inositol 
and its fit was assigned to the correct resonance, giving a minimal residue, the fitting result was accepted.

The LCModel software package (version 6.3-0C [31]) was used for postprocessing (e.g., eddy current correction and water scaling) and for automatic fitting of all ${ }^{1} \mathrm{H}-\mathrm{MR}$ spectra (i.e., two hippocampal voxels, ACC and PCC). The signal intensities of NAA, mI and glycine, and cholinecontaining compounds (tCho: $\mathrm{PCh}, \mathrm{GPCh}$, and free choline) were expressed relative to the intensity of creatine and phosphocreatine $(\mathrm{tCr})$. Additionally, water-referenced levels of NAA, mI, tCho, and $\mathrm{tCr}$, expressed in millimolar tissue concentration, and the ratio of NAA to mI were calculated. In the quantitative evaluation of the fitting results, a whole spectrum was rejected when the full-width halfmaximum was $>0.15 \mathrm{ppm}(18.5 \mathrm{~Hz})$ or the signal-to-noise ratio was $<5$, and only individual metabolite fits with a CRLB $<30 \%$ were considered reliable. Qualitatively, two spectroscopists (AR and MvdG) independently judged the original spectra and the fitting results by visual inspection according to a prespecified set of criteria.

T1-weighted MR images from one visit were segmented into gray matter (GM), white matter (WM), and CSF using automatic segmentation software (SPM8, Welcome Trust Centre for Neuroimaging, London, UK; VBM8, Structural Brain Mapping Group, Jena, Germany). For each subject, the whole-brain GM fraction (GM/[GM + $\mathrm{WM}+\mathrm{CSF}]$ ) was calculated and used as a prespecified confounder in supportive covariate analyses.

\section{Statistical analyses}

All statistical analyses were performed for the modified intention-to-treat (ITT) dataset, including all subjects who received at least one unit of the study product and had at least one MR measurement. The main statistical analyses of the MRS outcome parameters were also performed for the per-protocol (PP) population, which includes all subjects who had no major protocol deviations. All blood outcome parameters at week 4 were analyzed using analysis of covariance (ANCOVA) with between-subjects factors intervention group and sex, and with baseline measure as covariate.

All MRS outcome parameters at week 4 were analyzed using a predefined multilevel model with intervention group (test or control), sex, and brain region and its interaction with intervention group as fixed factors, considering brain region as a within-subject factor and adjusting for baseline. An unstructured variance-covariance matrix for brain region was selected. If the $p$ value for intervention group by brain region interaction was $<0.10$, ANCOVA models per brain region were used. If the $p$ value for the intervention by brain region interaction was $>0.10$, the interaction term was dropped from the model. For all fitted models, the influence diagnostics were used to explore the influence of different observations on the models. Analyses of the main ${ }^{31} \mathrm{P}$-MRS (PME, PDE, PME/PDE) and main
${ }^{1} \mathrm{H}$-MRS outcomes (NAA/tCr, mI/tCr, Cho/tCr, NAA/mI) were performed on partially unblinded data (using intervention group coded as $\mathrm{X}$ and $\mathrm{Y}$ ), allowing analysis of the intervention effect while keeping the statisticians blinded to group allocation. The primary statistical analyses of these main outcome parameters were repeated with adjustments for possible confounders (i.e., MMSE, age, education level [low, medium, high], GM fraction, and intake of cholinecontaining food [only for choline-related outcome parameters]).

Additionally, the following predefined supportive analyses were performed on the main ${ }^{31} \mathrm{P}-\mathrm{MRS}$ (PME, PDE, $\mathrm{PME} / \mathrm{PDE}$ ) and main ${ }^{1} \mathrm{H}-\mathrm{MRS}$ (NAA/tCr, $\mathrm{mI} / \mathrm{tCr}, \mathrm{Cho} /$ $\mathrm{tCr}, \mathrm{NAA} / \mathrm{mI}$ ) outcome parameters. First, the primary analyses described above were repeated using imputed baseline values when baseline values were missing. Thus, MRS outcome parameters at week 4 were analyzed using a predefined multilevel model with intervention group (test or control), sex, and brain region and its interaction with intervention group as fixed factors, considering brain region as a within-subject factor and adjusting for baseline, where missing baseline values were replaced by imputed baseline values (so-called multilevel model with imputation). Imputation of the baseline value was performed by a regression imputation using age, sex, and brain region. Second, linear mixed model analyses were conducted including the baseline value and the week 4 value of the outcome parameter in the outcome vector and including intervention group, sex, time, brain region, and two-way and three-way interactions between intervention group, brain region, and time as fixed factors, and a random intercept for time per subject. In this supportive model (so-called mixed model with time), the intervention effect is expressed by the interaction of time by intervention group. Third, the primary analyses were repeated for the one brain region for which most data were available. For all fitted models, the influence diagnostics were used to explore the influence of different observations on the models.

Statistical analyses were performed by both AR and ACY using SAS 9.2 and SAS 9.4 software, respectively (SAS Institute Inc., Cary, NC, USA). Statistical significance for the intervention effect was set at $p<0.05$ without correction for multiple testing.

\section{Results}

Subjects

Of the 40 subjects who were screened, 34 subjects were included and randomized to receive either the test or the control product. One subject dropped out of the study without MR measurements and prior to product dispensing, owing to unexpected claustrophobia, which was an exclusion criterion for the present study as described in the study protocol. Hence, 33 subjects were 
included in the modified ITT population. Major protocol deviations were present for four subjects: no MR measurements at week $4(n=2)$ or (suspected) double product intake prior to week 4 measurements $(n=2)$. Hence, 29 subjects were included in the PP population (for a flowchart, see Fig. 2). Subjects in the test $(n=16)$ and control $(n=17)$ groups (modified ITT population) were comparable with respect to baseline characteristics (Table 2). The test group reported 80 medical conditions in the medical history, as compared with 120 medical conditions in the control group. Adherence to the study product, according to diary entries, was high $(>96 \%)$ and equal in both groups.

\section{Safety analysis and concomitant medication}

In total, 27 adverse events (AEs) were reported, none of which were serious AEs. The number of subjects with at least one $\mathrm{AE}$ did not differ statistically between groups (test: 13 AEs in 8 subjects, control: 14 AEs in 7 subjects, $p=0.732$ by Fisher's exact test). A total of 15 of 33 subjects (45.5\% of the total study population) reported at least one AE. AEs that were most often reported for both study groups concerned gastrointestinal system disorders (i.e., abdominal pain, diarrhea, dyspepsia, nausea, vomiting). The majority of AEs were considered to be unrelated to the study product. Seven AEs (five in the test group and two in the control group) had a relationship to the study product ("possibly" or "probably"), and were all of gastrointestinal nature (i.e., diarrhea, dyspepsia, nausea, vomiting). Regarding the use of
Table 2 Subject characteristics

\begin{tabular}{|c|c|c|}
\hline & Test $(n=16)$ & Control $(n=17)$ \\
\hline Age, years & $74.7(4.8)$ & $72.7(8.2)$ \\
\hline Male sex, $n(\%)$ & $7(44 \%)$ & $7(41 \%)$ \\
\hline MMSE score & $23.0(2.1)$ & $23.2(1.8)$ \\
\hline Time since diagnosis, months & $1.7[0.4-15.3]$ & $1.4[0.2-10.2]$ \\
\hline $\mathrm{BMI}, \mathrm{kg} / \mathrm{m}^{2}$ & $24.2(3.8)$ & $26.7(3.3)$ \\
\hline \multicolumn{3}{|l|}{ Educational level, $n$ (\%) } \\
\hline Low & $6(37.5 \%)$ & $4(23.5 \%)$ \\
\hline Medium & $8(50.0 \%)$ & $9(52.9 \%)$ \\
\hline High & $2(12.5 \%)$ & $4(23.5 \%)$ \\
\hline Study product compliance & $96.4 \%(4.8)$ & $99.0 \%(1.6)$ \\
\hline
\end{tabular}

concomitant medication (other than $\mathrm{AD}$ medication), 62.5\% of subjects in the test group and $82.4 \%$ of the control group used concomitant medication until the 4-week intervention period. After the intervention period, 4 of 16 subjects in the test group and 8 of 17 subjects in the control group started $\mathrm{AD}$ medication (galantamine, memantine, or rivastigmine).

\section{Nutritional blood markers}

Levels of uridine, choline, and vitamin E in plasma, as well as percentages of DHA, EPA, and total long-chain

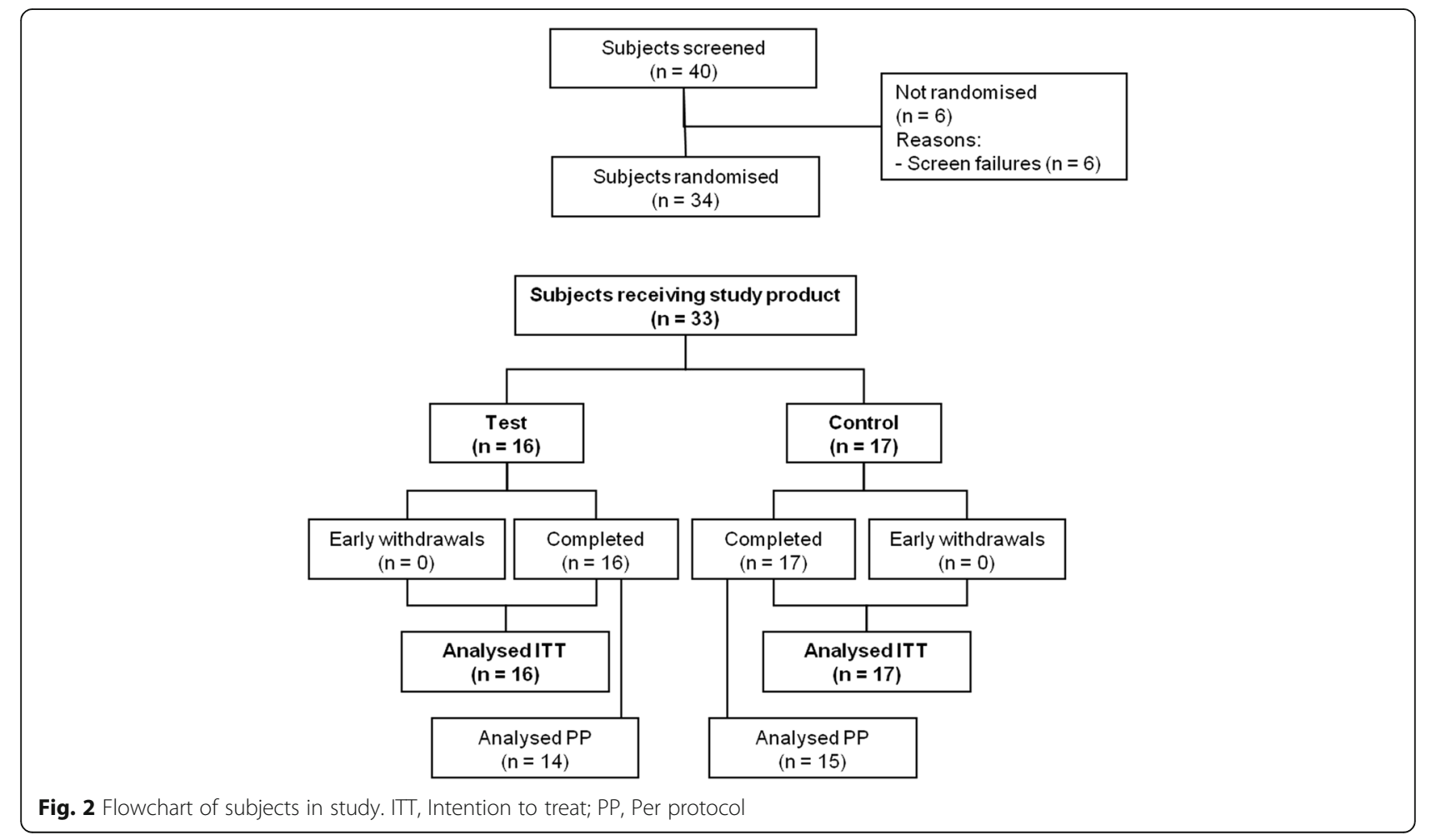


polyunsaturated fatty acids in plasma and in total fatty acids in erythrocyte membrane, were higher at week 4 in the test group than in the control group (all $p<0.001$ ). Homocysteine levels were lower after 4 weeks of test product compared with the control product $(p=0.006)$. Docosapentaenoic acid in plasma or erythrocyte membrane was not significantly different at week 4 between the groups $(p=0.628$ and $p=0.840$, respectively). For details, see Table 3.

\section{MRS outcomes}

Good-quality ${ }^{31} \mathrm{P}$-MR and ${ }^{1} \mathrm{H}$-MR spectra were obtained from all ROIs (Fig. 1b and d). If no significant interactions between intervention group and brain region were observed in the statistical analyses, only main intervention group effects are reported. Furthermore, the results from the covariate analyses are reported only if the potential confounders substantially changed the intervention effect. Because the ITT and PP analyses yielded the same findings, only the ITT analyses are reported.

\section{Primary ${ }^{31}$ P-MRS outcomes}

A significant intervention effect showing higher PME/ PDE at week 4 in the test group than in the control group was found using the primary statistical analysis approach (i.e., predefined multilevel model) (least squares [LS] mean \pm SEM test: $1.35 \pm 0.06$, control: 1.17 $\pm 0.06, p=0.005$ ) (Fig. 3). Visual inspection of the data and the results from the supportive model indicated that PME/PDE increased over time in the test group and decreased over time in the control group (see Additional file 1: Table S1, model 2).
No differences between groups were observed regarding the levels of PME LS mean \pm SEM at week 4 (test: $12.05 \pm$ 0.19 , control: $11.93 \pm 0.19, p=0.628$ ) or regarding levels of PDE (LS mean \pm SEM at week 4, test: $9.79 \pm 0.23$, control: $9.89 \pm 0.22, p=0.702)$. For the results from the supportive models, see Additional file 1: Table S1.

\section{Other ${ }^{31} \mathrm{P}-\mathrm{MRS}$ outcomes}

The PEth/GPEth ratio was higher in the test group than in the control group (LS mean \pm SEM test: $2.02 \pm 0.09$, control: $1.80 \pm 0.09$, LS mean difference [95\% CI] 0.22 [ -0.04 to 0.48], $p=0.091$ ) (Fig. 4). There were no significant differences between groups regarding the $\mathrm{PCh} / \mathrm{GPCh}$ ratio; levels of PEth, PCh, GPEth, GPCh, PCr, Pi, ATP, or nicotinamide adenine dinucleotide; or $\mathrm{pH}$ (all $p>0.05$ ).

\section{${ }^{1} \mathrm{H}$-MRS outcomes}

Using the primary statistical analysis approach, a significant interaction with brain region was found for tCho/ $\mathrm{tCr}$, leading to analyses per brain region. These showed that $\mathrm{tCho} / \mathrm{tCr}$ at week 4 was higher in the test group than in the control group in the ACC (LS mean \pm SEM test: $0.302 \pm 0.015$, control: $0.258 \pm 0.016, p=0.068)$ and in the HR (LS mean \pm SEM test: $0.287 \pm 0.004$, control: $0.264 \pm 0.004, p=0.003$ ), but not in the HL and PCC (both $p>0.05$ ) (Fig. 5). For the results from the supportive models, see Additional file 1: Table S2.

For absolute levels of tCho, a significant intervention effect indicated that levels of tCho were higher at week 4 in the test group than in the control group (LS mean \pm SEM test: $1.94 \pm 0.05$, control: $1.83 \pm 0.05, p=0.018$; LS mean difference $[95 \% \mathrm{CI}] 0.11$ [0.02 to 0.20$], p=0.018$ ).

Table 3 Intervention effect on nutritional blood markers and homocysteine

\begin{tabular}{|c|c|c|c|c|}
\hline \multirow[t]{2}{*}{ Blood outcome parameter } & \multicolumn{2}{|c|}{ Mean estimated value at week 4} & \multirow{2}{*}{$\begin{array}{l}\text { Difference between } \\
\text { groups at week } 4\end{array}$} & \multirow{2}{*}{$\begin{array}{l}p \text { Value for } \\
\text { intervention effec }\end{array}$} \\
\hline & Test $(n=16)$ & Control $(n=17)$ & & \\
\hline \multicolumn{5}{|l|}{ Fatty acids in the erythrocyte membrane } \\
\hline $\mathrm{DHA}, \%$ & 4.9 & 2.8 & $2.1(1.84$ to 2.30$)$ & $<0.001$ \\
\hline EPA, \% & 1.7 & 0.8 & 0.8 (0.69 to 0.98$)$ & $<0.001$ \\
\hline DPA, \% & 1.6 & 1.6 & $0.014(-0.12$ to 0.15$)$ & 0.840 \\
\hline LC-PUFA (DHA + EPA + DPA), \% & 8.2 & 5.3 & $2.9(2.51$ to 3.24$)$ & $<0.001$ \\
\hline \multicolumn{5}{|l|}{ Fatty acids in blood plasma } \\
\hline $\mathrm{DHA}, \%$ & 4.4 & 1.5 & 2.9 (2.59 to 3.12$)$ & $<0.001$ \\
\hline EPA, \% & 2.1 & 0.8 & $1.3(1.10$ to 1.51$)$ & $<0.001$ \\
\hline DPA, \% & 0.50 & 0.49 & $0.008(-0.03$ to 0.04$)$ & 0.628 \\
\hline LC-PUFA (DHA + EPA + DPA), \% & 7.0 & 2.8 & 4.2 (3.79 to 4.53 ) & $<0.001$ \\
\hline Choline levels in blood plasma, $\mu \mathrm{M}$ & 14.4 & 9.0 & 5.4 (3.39 to 7.47$)$ & $<0.001$ \\
\hline Vitamin E levels in blood plasma, $\mu \mathrm{M}$ & 50.9 & 39.6 & $11.4(6.77$ to 15.96$)$ & $<0.001$ \\
\hline Homocysteine levels in blood plasma, $\mu \mathrm{M}$ & 9.8 & 12.4 & $-2.6(-4.31$ to -0.82$)$ & 0.006 \\
\hline Uridine levels in blood plasma, $\mu \mathrm{M}$ & 14.8 & 2.6 & $12.2(7.24$ to 17.11$)$ & $<0.001$ \\
\hline
\end{tabular}

Abbreviations: DHA Docosahexaenoic acid, EPA Eicosapentaenoic acid, DPA Docosapentaenoic acid, LC-PUFA Long-chain polyunsaturated fatty acids The $95 \% \mathrm{Cl}$ is presented in parentheses 


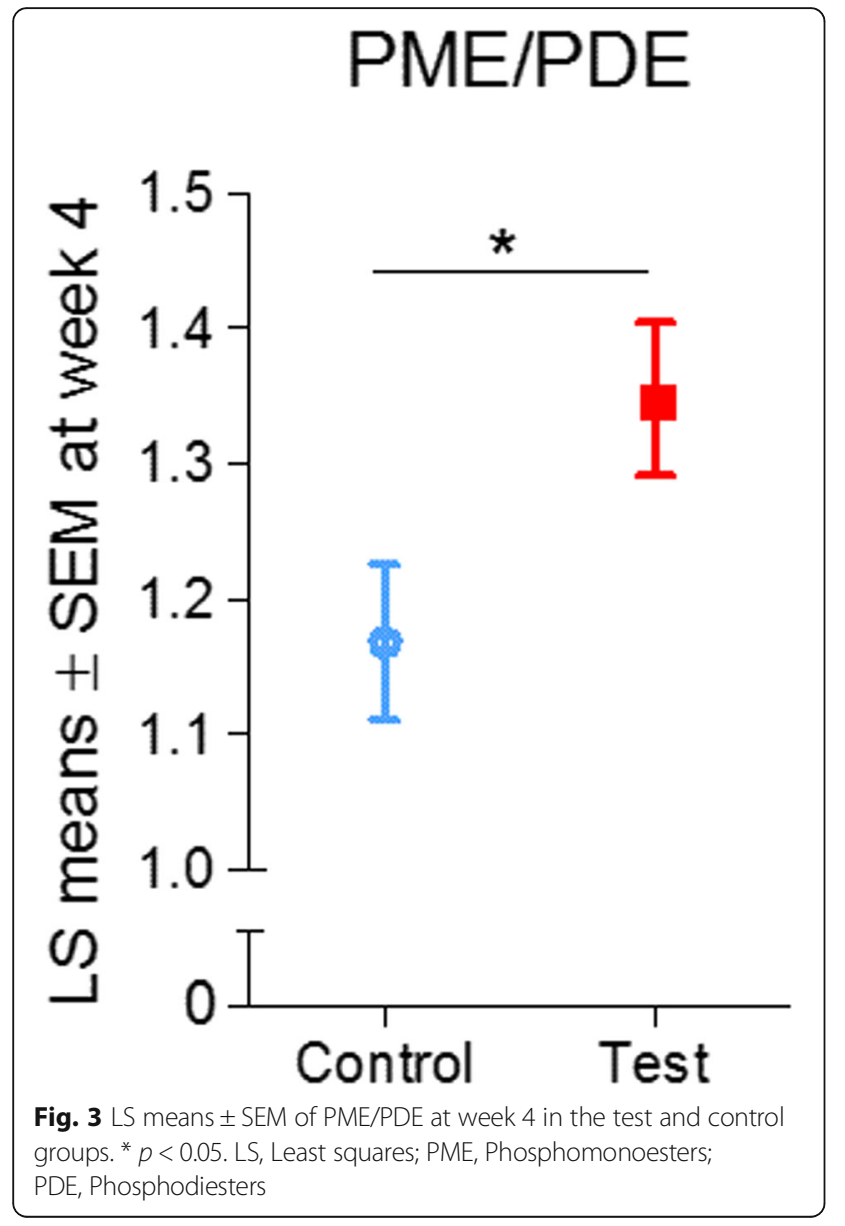

There were no significant differences between groups regarding relative (i.e., relative to $\mathrm{tCr}$ ) or absolute (i.e., water-referenced) levels of NAA or $\mathrm{mI}$ on the ratio NAA/ $\mathrm{mI}$ or regarding absolute levels of tCre (all $p>0.05$ ).

\section{Discussion}

In this study, we investigated whether the medical food Souvenaid, containing nutritional precursors and cofactors for phospholipid membrane formation (i.e., FC), influenced brain phospholipid metabolism in mild AD. The observed effects indicate that this specific multinutrient combination not only raises circulating levels of phospholipid precursors after 4 weeks but also affects the balance between brain metabolites of phospholipid formation and breakdown in patients with mild AD. In addition, levels of tCho were higher after the intervention in comparison with the control product, whereas metabolic measures of neural integrity, gliosis, and energy metabolism were not significantly affected.

There is strong ex vivo evidence that in the AD brain, phospholipid content is decreased [3], phospholipid membrane composition is altered [3, 32], and phospholipid anabolic and catabolic processes are disturbed [7, 33], and
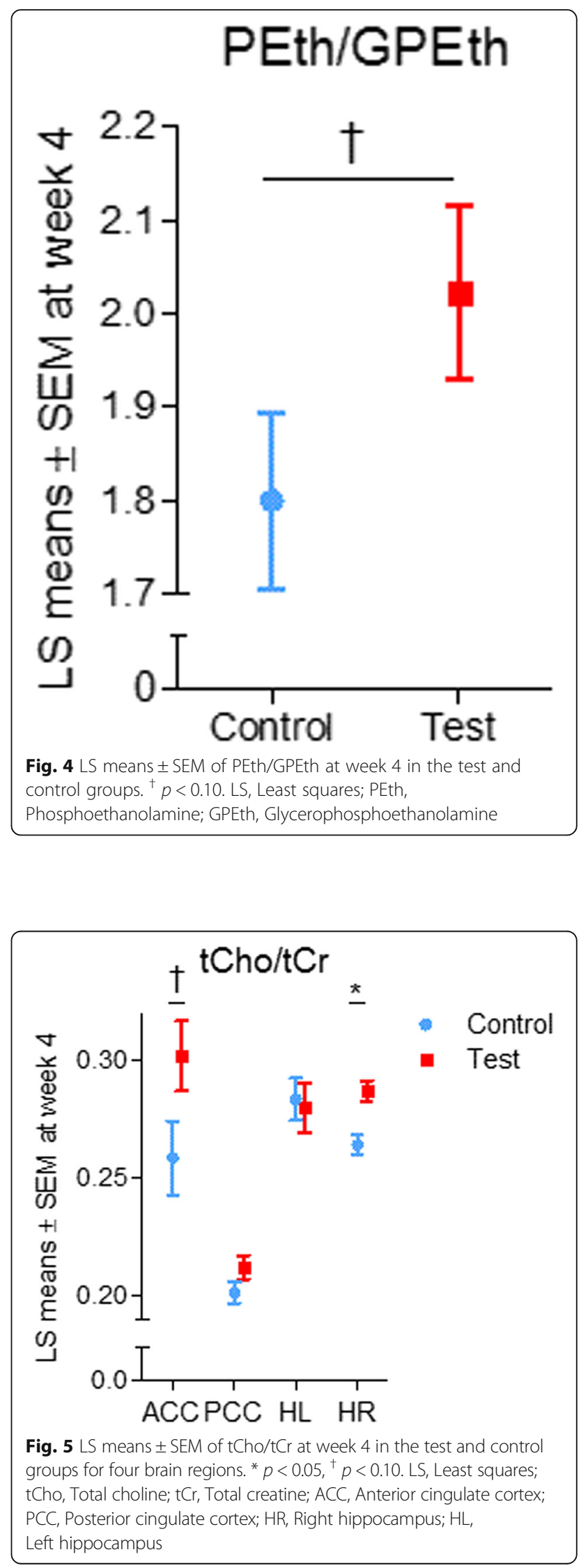
a tight link of these changes with synaptic loss and synaptic dysfunction is presumed [32, 34]. The dependence of the unsaturated, low-affinity enzymes in the phospholipid synthesis pathway on substrate availability offers the opportunity to support this process by providing those substrates that are rate-limiting. Short-term supplementation with this specific multinutrient combination increases plasma and erythrocyte levels of nutritional precursors and cofactors for phospholipid membrane formation in patients with mild $A D$, which confirms and extends previous studies [27], and thus may alleviate preexisting nutritional deficiencies [14]. Because several mechanisms have been described that move key nutrients across the bloodbrain barrier [9], the nutrients in the present investigation, or their metabolites, are expected to reach the brain. Accordingly, we observed a significantly increased PME/PDE ratio, which is considered to reflect the ratio of phospholipid anabolites over catabolites [22, 35], across four brain regions with 4 weeks of daily use of this multinutrient combination in mild AD. This indicates that the nutrients exert their effect on the brain's phospholipid metabolism, in line with the hypothesized mode of action of this multinutrient combination. The observed changes in phospholipid metabolism may promote neuronal membrane formation and may stimulate dendritic spine formation, as was shown previously in animal studies $[8,9,11]$. The remodeling of phospholipid membranes in the brain will probably continue with prolonged intake, and thus continuous intake is needed for long-term effects on synapse formation. This may underlie the effects that this intervention has on memory in patients with mild $\operatorname{AD}[19,20]$. Future research is needed to confirm this hypothesis, as well as to investigate the actual impact of the changes in phospholipid metabolism on changes in synaptic function, functional connectivity and brain structural volumes in $\mathrm{AD}$ pathology, or on $\mathrm{AD}$ progression. Imaging techniques such as ${ }^{18} \mathrm{~F}$-fluorodeoxyglucose positron emission tomography and structural MRI are currently used in ongoing randomized controlled trials to further explore whether this multinutrient combination affects synaptic function and structural brain volumes [36].

It was observed that the significantly increased PME/PDE ratio was driven by both an increased PME/PDE ratio in the active group and a decreased PME/PDE ratio in the control group. The decreased PME/PDE ratio in the control group was not expected over the course of 4 weeks of follow-up. The clinical significance of this decrease with respect to disease progression is currently unclear.

Because the PME/PDE ratio changed significantly but individual and total phosphomonoesters (PEth, $\mathrm{PCh}$, and PME) and phosphodiesters (GPEth, GPCh, and PDE) did not, it cannot be established whether phospholipid formation was increased, breakdown was decreased, or both. In addition, the literature is inconclusive on in vivo levels of these metabolites in AD because increased, decreased, and unaltered levels of PME and PDE have been observed in comparison with cognitively normal controls [37-42]. However, the effect of this multinutrient combination on brain phospholipid metabolism appears to be driven mainly by the ethanolamine pathway because PEth/ GPEth, but not PCh/GPCh, showed a trend toward an increase. This is in line with other studies that show that oral phospholipid precursors (pyrimidines and choline) in healthy populations $[25,26,43]$ predominantly affect the ethanolamine pathway. However, in the present study, both relative and absolute tCho levels were higher at follow-up in the group receiving the multinutrient intervention than in the control group, indicating an effect on phosphocholine metabolism as well. The tCho signal reflects the choline-containing compounds $\mathrm{PCh}, \mathrm{GPCh}$, and free choline, and while both PCh and GPCh were measured separately with ${ }^{31} \mathrm{P}$-MRS, no increase was detected in either one. This discrepancy may have arisen because the measured ${ }^{1} \mathrm{H}$-MRS and ${ }^{31} \mathrm{P}$-MRS brain volumes differed, but supportive statistical models could not confirm the significant alterations of tCho after a 4-week intervention, warranting cautious interpretation. The analyses did reveal that changes in tCho may not be anatomically uniform, because the most prominent effects were observed in the ACC and HR. Because choline levels also increase in plasma, it is important to rule out that the measured increase in brain choline does not just reflect the vascular component of the measured volume. Using the available data, it was estimated that the change in plasma levels would constitute only about $0.2 \%$ of the change observed in brain choline, even when assuming that all plasma choline is MR-visible and estimating the vascular contribution at a liberal $5 \%$.

Reduced levels of NAA and increased levels of $\mathrm{mI}$ have been shown consistently in $\mathrm{AD}$ [44], and it might have been expected that this multinutrient combination would decelerate the changes in these measures of neural integrity and gliosis. Although uridine and DHA were found to stimulate neurite outgrowth $[45,46]$, and DHA is known to have anti-inflammatory effects in addition to its role in the phospholipid synthesis pathway [47], no group differences after 4 weeks of intervention were observed regarding relative or absolute levels of NAA or mI, nor were differences observed regarding the NAA $/ \mathrm{mI}$ ratio. However, the normal rate of change over 4 weeks' time may be limited, such that a deceleration effect of the intervention is not yet discernible. Although 4 weeks of intervention did affect phospholipid metabolism, this period may have been too short to observe an effect on metabolic measures of neural integrity and gliosis. Alternatively, it may be that the damage that these metabolites reflect is already irreversible at this stage of the disease. 
The findings of this study may be limited by the modest sample size. Although there was sufficient power to detect an effect on the PME/PDE ratio, one of the main outcome measures, it was not possible to ascribe the increase in this ratio to increased PME or decreased PDE. Moreover, MR spectra were not always available or of sufficient quality from all investigated brain regions. Finally, a longer intervention duration may have led to more robust findings and more pronounced secondary outcomes.

\section{Conclusions}

This exploratory, double-blind, randomized controlled study shows that the medical food Souvenaid affects phospholipid metabolism across multiple brain regions in mild $\mathrm{AD}$ after only 4 weeks. This could lead to increased neuronal membrane formation, which would support the hypothesized mode of action of this multinutrient intervention. Larger and longer randomized controlled trials are needed to determine long-term effects on phospholipid formation, synaptic function, and cognition in persons with and at risk for $\mathrm{AD}$.

\section{Additional file}

Additional file 1: Supplement to 'A. Rijpma, M. van der Graaf, M.M. Lansbergen, O. Meulenbroek, A. Cetinyurek-Yavuz, J.W. Sijben, A. Heerschap, and M.G.M. Olde Rikkert, The medical food Souvenaid affects brain phospholipid metabolism in mild Alzheimer's disease: results from a randomized controlled trial'. (PDF $614 \mathrm{~kb}$ )

\begin{abstract}
Abbreviations
${ }^{1} \mathrm{H} /{ }^{31}$ P-MRS: Proton/phosphorus magnetic resonance spectroscopy; ACC: Anterior cingulate cortex; AD: Alzheimer's disease; AE: Adverse event; ANCOVA: Analysis of covariance; ATP: Adenosine triphosphate; BMI: Body mass index; Cr: Creatine; CRLB: Cramér-Rao lower bound; CSF: Cerebrospinal fluid; DHA: Docosahexaenoic acid; DPA: Docosapentaenoic acid; EPA: Eicosapentaenoic acid; FC: Fortasyn Connect; FOV: Field of view; GDS: Geriatric Depression Scale; GM: Gray matter;

GPCh: Glycerophosphocholine; GPEth: Glycerophosphoethanolamine; HL: Left hippocampus; HR: Right hippocampus; ITT: Intention to treat; LC-PUFA: Longchain polyunsaturated fatty acid; LS: Least squares; ml: Myo-inositol; MMSE: Mini Mental State Examination; MR: Magnetic resonance; MRI: Magnetic resonance imaging; MRS: Magnetic resonance spectroscopy; MRSI: 3D magnetic resonance spectroscopic imaging; NAA: N-acetylaspartate; PC: Phosphatidylcholine; PCC: Posterior cingulate cortex;

PCh: Phosphocholine; PCr: Phosphocreatine; PDE: Phosphodiester; PE: Phosphatidylethanolamine; PEth: Phosphoethanolamine; Pi: Inorganic phosphate; PME: Phosphomonoester; PP: Per protocol; PRESS: Point resolved spectroscopy; ROI: Region of interest; RSC: Retrosplenial cortex; SLASER: Semilocalization by adiabatic selective refocusing; TA: Acquisition time; tCho: Total choline; tCr: Total creatine; TE: Echo time; TR: Repetition time; UMP: Uridine monophosphate; WM: White matter
\end{abstract}

\section{Acknowledgements}

We thank all participants and their caregivers for participating in this study. We are grateful to the centers that referred patients to participate in this study (Canisius Wilhelmina Ziekenhuis and Ziekenhuis Rivierenland Tiel). We are indebted to William van Aalst for his help in recruitment of participants, to Rianne de Heus for her assistance during data acquisition and analysis, and to Jack van Asten and Miriam Lagemaat for their help in developing the acquisition and postprocessing protocols (WvA and RdH: Department of Geriatric Medicine, Radboud University Medical Center; JvA and ML: Department of Radiology and Nuclear Medicine, Radboud University Medical Center).

\section{Funding}

This study was sponsored by Nutricia Research, Nutricia Advanced Medical Nutrition.

\section{Availability of data and materials}

The data that support the findings of this study are available from Nutricia Research, Nutricia Advanced Medical Nutrition, but restrictions apply to the availability of these data, which were used under license for the present study and so are not publicly available. Data are available, however, from the authors upon reasonable request and with permission of Nutricia Research, Nutricia Advanced Medical Nutrition.

\section{Authors' contributions}

$\mathrm{AH}, \mathrm{MGMOR}$, JWS, and MML conceived of and designed the study. AR, MvdG, OM, and ACY acquired and analyzed data. AR drafted the manuscript. All authors edited, read, and approved the final manuscript.

\section{Ethics approval and consent to participate}

Written informed consent was obtained from all patients and their informal caregivers. The local ethics committee (Commissie Mensgebonden Onderzoek Arnhem Nijmegen; 2011-325) reviewed and approved the protocol.

\section{Competing interests}

AR, MvdG, OM, AH, and MGMOR declare that they have no competing interests. MML, ACY, and JWS are employees of Nutricia Research, Nutricia Advanced Medical Nutrition. MML is mentioned as inventor on patent application PCT/NL2015/050064 = WO2015/115898, which is owned by Nutricia

\section{Publisher's note}

Springer Nature remains neutral with regard to jurisdictional claims in published maps and institutional affiliations.

\section{Author details}

${ }^{1}$ Department of Geriatric Medicine, Radboud University Medical Center, P.O. Box 9101, 6500 HB Nijmegen, The Netherlands. ${ }^{2}$ Radboudumc Alzheimer Center, Donders Institute for Brain, Cognition and Behavior, Radboud University Medical Center, Nijmegen, The Netherlands. ${ }^{3}$ Department of Radiology and Nuclear Medicine, Radboud University Medical Center, Nijmegen, The Netherlands. ${ }^{4}$ Department of Pediatrics, Radboud University Medical Center, Nijmegen, The Netherlands. ${ }^{5}$ Nutricia Research, Nutricia Advanced Medical Nutrition, Utrecht, The Netherlands.

Received: 15 March 2017 Accepted: 10 July 2017

Published online: 26 July 2017

\section{References}

1. Terry RD, Masliah E, Salmon DP, Butters N, DeTeresa R, Hill R, et al. Physical basis of cognitive alterations in Alzheimer's disease: synapse loss is the major correlate of cognitive impairment. Ann Neurol. 1991;30:572-80.

2. Selkoe DJ. Alzheimer's disease is a synaptic failure. Science. 2002;298:789-91.

3. Nitsch RM, Blusztajn JK, Pittas AG, Slack BE, Growdon JH, Wurtman RJ. Evidence for a membrane defect in Alzheimer disease brain. Proc Natl Acad Sci U S A. 1992;89:1671-5.

4. Pettegrew JW, Panchalingam K, Hamilton RL, McClure RJ. Brain membrane phospholipid alterations in Alzheimer's disease. Neurochem Res. 2001;26: 771-82.

5. Sastry PS. Lipids of nervous tissue: composition and metabolism. Prog Lipid Res. 1985:24:69-176.

6. Kennedy EP, Weiss SB. The function of cytidine coenzymes in the biosynthesis of phospholipides. J Biol Chem. 1956;222:193-214.

7. Ross BM, Moszczynska A, Erlich J, Kish SJ. Phospholipid-metabolizing enzymes in Alzheimer's disease: increased lysophospholipid acyltransferase activity and decreased phospholipase $A_{2}$ activity. J Neurochem. 1998;70: 786-93.

8. $\quad$ van Wijk N, Broersen LM, de Wilde MC, Hageman RJ, Groenendijk M, Sijben JW, et al. Targeting synaptic dysfunction in Alzheimer's disease by administering a specific nutrient combination. J Alzheimers Dis. 2014;38: 459-79.

9. Wurtman RJ, Cansev M, Sakamoto T, Ulus IH. Use of phosphatide precursors to promote synaptogenesis. Annu Rev Nutr. 2009;29:59-87. 
10. Cansev M, Wurtman RJ, Sakamoto T, Ulus IH. Oral administration of circulating precursors for membrane phosphatides can promote the synthesis of new brain synapses. Alzheimers Dement. 2008;4(1 Suppl 1):S153-68.

11. Sakamoto T, Cansev M, Wurtman RJ. Oral supplementation with docosahexaenoic acid and uridine-5'-monophosphate increases dendritic spine density in adult gerbil hippocampus. Brain Res. 2007;1182:50-9.

12. Wurtman RJ, Ulus IH, Cansev M, Watkins CJ, Wang L, Marzloff G. Synaptic proteins and phospholipids are increased in gerbil brain by administering uridine plus docosahexaenoic acid orally. Brain Res. 2006;1088:83-92.

13. Lopes da Silva S, Vellas B, Elemans S, Luchsinger J, Kamphuis P, Yaffe K, et al. Plasma nutrient status of patients with Alzheimer's disease: systematic review and meta-analysis. Alzheimers Dement. 2014;10:485-502.

14. Olde Rikkert MG, Verhey FR, Sijben JW, Bouwman FH, Dautzenberg PL, Lansink $M$, et al. Differences in nutritional status between very mild Alzheimer's disease patients and healthy controls. J Alzheimers Dis. 2014;41:261-71.

15. de Wilde MC, Vellas B, Girault E, Cetinyurek-Yavuz A, Sijben JW. Lower brain and blood nutrient status in Alzheimer's disease: results from meta-analyses. Alzheimers Dement. doi:10.1016/j.trci.2017.06.002.

16. Jansen D, Zerbi V, Arnoldussen IC, Wiesmann M, Rijpma A, Fang XT, et al. Effects of specific multi-nutrient enriched diets on cerebral metabolism, cognition and neuropathology in AßPPswe-PS1dE9 mice. PLoS One. 2013;8: e75393.

17. Wiesmann M, Jansen D, Zerbi V, Broersen LM, Garthe A, Kiliaan AJ. Improved spatial learning strategy and memory in aged Alzheimer ABPPswe/PS1dE9 mice on a multi-nutrient diet. J Alzheimers Dis. 2013;37:233-45.

18. Cansev M, van Wijk N, Turkyilmaz M, Orhan F, Sijben JWC, Broersen LM. Specific multi-nutrient enriched diet enhances hippocampal cholinergic transmission in aged rats. Neurobiol Aging. 2015;36:344-51.

19. Scheltens P, Kamphuis PJGH, Verhey FRJ, Rikker MGMO, Wurtman RJ, Wilkinson D, et al. Efficacy of a medical food in mild Alzheimer's disease: a randomized, controlled trial. Alzheimers Dement. 2010;6:1-10.e1.

20. Scheltens P, Twisk JWR, Blesa R, Scarpini E, von Arnim CAF, Bongers A, et al, Efficacy of Souvenaid in mild Alzheimer's disease: results from a randomized, controlled trial. J Alzheimers Dis. 2012;31:225-36.

21. de Waal H, Stam CJ, Lansbergen MM, Wieggers RL, Kamphuis PJ, Scheltens $P$, et al. The effect of Souvenaid on functional brain network organisation in patients with mild Alzheimer's disease: a randomised controlled study. PLoS One. 2014;9:e86558.

22. Martin WRW. MR spectroscopy in neurodegenerative disease. Mol Imaging Biol. 2007:9:196-203.

23. Oz G, Alger JR, Barker PB, Bartha R, Bizzi A, Boesch C, et al. Clinical proton MR spectroscopy in central nervous system disorders. Radiology. 2014;270: 658-79.

24. McKhann GM, Knopman DS, Chertkow H, Hyman BT, Jack Jr CR, Kawas CH, et al. The diagnosis of dementia due to Alzheimer's disease: recommendations from the National Institute on Aging-Alzheimer's Association workgroups on diagnostic guidelines for Alzheimer's disease. Alzheimers Dement. 2011;7:263-9.

25. Agarwal N, Sung YH, Jensen JE, daCunha G, Harper D, Olson D, et al. Shortterm administration of uridine increases brain membrane phospholipid precursors in healthy adults: a 31-phosphorus magnetic resonance spectroscopy study at 4T. Bipolar Disord. 2010;12:825-33.

26. Silveri MM, Dikan J, Ross AJ, Jensen JE, Kamiya T, Kawada Y, et al. Citicoline enhances frontal lobe bioenergetics as measured by phosphorus magnetic resonance spectroscopy. NMR Biomed. 2008;21:1066-75.

27. Rijpma A, Meulenbroek O, van Hees AM, Sijben JW, Vellas B, Shah RC, et al, Effects of Souvenaid on plasma micronutrient levels and fatty acid profiles in mild and mild-to-moderate Alzheimer's disease. Alzheimers Res Ther. 2015;7:51.

28. Pohmann R, von Kienlin M. Accurate phosphorus metabolite images of the human heart by 3D acquisition-weighted CSI. Magn Reson Med. 2001;45:817-26.

29. de Graaf RA. In vivo NMR spectroscopy: principles and techniques. 2nd ed. Chichester: Wiley; 2007

30. Petroff OAC, Prichard JW, Behar KL, Alger JR, den Hollander JA, Shulman RG. Cerebral intracellular $\mathrm{pH}$ by ${ }^{31} \mathrm{P}$ nuclear magnetic resonance spectroscopy. Neurology. 1985;35:781-8.

31. Provencher SW. Estimation of metabolite concentrations from localized in vivo proton NMR spectra. Magn Reson Med. 1993;30:672-9.

32. Martín V, Fabelo N, Santpere G, Puig B, Marín R, Ferrer l, et al. Lipid alterations in lipid rafts from Alzheimer's disease human brain cortex. Alzheimers Dis. 2010;19:489-502.
33. Ross BM, Moszczynska A, Blusztajn JK, Sherwin A, Lozano A, Kish SJ. Phospholipid biosynthetic enzymes in human brain. Lipids. 1997:32:351-8.

34. Pomponi M, Bria P, Pomponi M. Is Alzheimer's disease a synaptic disorder? J Alzheimers Dis. 2008;13:39-47.

35. Pettegrew JW. Molecular insights into Alzheimer's disease. Ann N Y Acad Sci. 1989:568:5-28.

36. Scheltens NME, Kuyper IS, Boellaard R, Barkhof F, Teunissen CE, Broersen LM et al. Design of the NL-ENIGMA study: exploring the effect of Souvenaid on cerebral glucose metabolism in early Alzheimer's disease. Alzheimers Dement (N Y). 2016;2:233-40.

37. Smith CD, Pettigrew LC, Avison MJ, Kirsch JE, Tinkhtman AJ, Schmitt FA, et al. Frontal lobe phosphorus metabolism and neuropsychological function in aging and in Alzheimer's disease. Ann Neurol. 1995;38:194-201.

38. Brown GG, Garcia JH, Gdowski JW, Levine SR, Helpern JA. Altered brain energy metabolism in demented patients with multiple subcortical ischemic lesions: working hypotheses. Arch Neurol. 1993;50:384-8.

39. Bottomley PA, Cousins JP, Pendrey DL, Wagle WA, Hardy CJ, Eames FA, et al Alzheimer dementia: quantification of energy metabolism and mobile phosphoesters with P-31 NMR spectroscopy. Radiology. 1992;183:695-9.

40. Pettegrew JW, Panchalingam K, Klunk WE, McClure RJ, Muenz LR. Alterations of cerebral metabolism in probable Alzheimer's disease: a preliminary study. Neurobiol Aging. 1994;15:117-32.

41. Forlenza OV, Wacker P, Nunes PV, Yacubian J, Castro CC, Otaduy MCG, et al. Reduced phospholipid breakdown in Alzheimer's brains: a ${ }^{31} \mathrm{P}$ spectroscopy study. Psychopharmacology (Berl). 2005;180:359-65.

42. Mandal PK, Akolkar $\mathrm{H}$, Tripathi M. Mapping of hippocampal pH and neurochemicals from in vivo multi-voxel ${ }^{31} \mathrm{P}$ study in healthy normal young male/female, mild cognitive impairment, and Alzheimer's disease. J Alzheimers Dis. 2012;31 Suppl 3:S75-86

43. Babb SM, Wald LL, Cohen BM, Villafuerte RA, Gruber SA, Yurgelun-Todd DA, et al. Chronic citicoline increases phosphodiesters in the brains of healthy older subjects: an in vivo phosphorus magnetic resonance spectroscopy study. Psychopharmacology (Berl). 2002:161:248-54.

44. Firbank MJ, Harrison RM, O'Brien JT. A comprehensive review of proton magnetic resonance spectroscopy studies in dementia and Parkinson's disease. Dement Geriatr Cogn. 2002;14:64-76.

45. Pooler AM, Guez DH, Benedictus R, Wurtman RJ. Uridine enhances neurite outgrowth in nerve growth factor-differentiated PC12. Neuroscience. 2005; 134:207-14.

46. Calderon F, Kim HY. Docosahexaenoic acid promotes neurite growth in hippocampal neurons. J Neurochem. 2004;90:979-88. A published erratum appears in J Neurochem. 2004;90:1540.

47. Farooqui AA, Horrocks LA, Farooqui T. Modulation of inflammation in brain: a matter of fat. J Neurochem. 2007:101:577-99.

\section{Submit your next manuscript to BioMed Central and we will help you at every step:}

- We accept pre-submission inquiries

- Our selector tool helps you to find the most relevant journal

- We provide round the clock customer support

- Convenient online submission

- Thorough peer review

- Inclusion in PubMed and all major indexing services

- Maximum visibility for your research

Submit your manuscript at www.biomedcentral.com/submit 